\title{
Prevalência de Dislipidemias na Demanda Laboratorial de Três Diferentes Prestadores de Assistência
}

\author{
Ines Lessa, João Lycio Conceição, Levi Mirabeau, Joabe Carneiro, Joamar M elo, Viviane O liveira, \\ Joel Pinheiro, Fábio Meireles, Joaquim Reis N eto, Felipe Reis, Rogério Gouvea, Marcelo Couto, \\ Marlene Rosa O liveira, Silvânia Souza
}

Salvador, BA

\begin{abstract}
Objetivo - Detectar diferenças nas prevalências das dislipidemias em adultos da demanda laboratorial de três diferentes prestadores de assistência médica, em Salvador, 1995.

Métodos - Estudo seccional, clínico epidemiológico, baseado em resultados das dosagens de lípides séricos de uma amostra probabilística de 25\% dos 104 laboratórios que usavam o mesmo método de dosagem e o mesmo padrão para controle de qualidade. O critério de dislipidemia foi o do II Consenso Brasileiro de Dislipidemias para adultos. Os resultados foram agrupados conforme procedência: Sistema Único de Saúde (SUS), convênios (C) e seguros privados + particulares (SP). Análise: prevalência com intervalos de confiança (IC) a 95\%, eteste do $\chi^{2}$.

Resultados - Dos 5.464 adultos com menção da procedência, $14,8 \%$ foram do SUS, $31,6 \%$ dos Ce 53,6\% dos $S+P$. As mais elevadas prevalências de hipercolesterolemia - 28,0\% (IC 24,0;32,0) e de LDL-c elevado$30,4 \%$ (IC 27,6; 33,2) foram detectadas no grupo C. No grupo $S+P$ concentraram-se as maiores prevalências de HDL-c anormal - 12,3\% (IC 10,0; 13,8) e hipertrigliceridemia - 17,8\% (IC 16,3; 19,3). Quase todas as diferenças entre as prevalências foram estatisticamente significantes, favoráveis às taxas mais baixas do SUS.

Conclusão - O melhor perfil lipídico do grupo SUS sugere diferenças sociais nas prevalências das dislipidemias. Para os três prestadores de assistência, a prevalência do HDL-c <35mg/dL foi baixa quando comparada às das demais dislipidemias. Os resultados podem ser inferidos para a prática médica dos respectivos prestadores de assistência e indicam a necessidade de informação e de orientação dos usuários para redução das dislipidemias e melhoria do estilo de vida.
\end{abstract}

Palavras-chave: dislipidemia, prevalência, prestadores de assistência

\section{Prevalence of Dyslipidemias in Adult Ambu- latory Laboratory Tests from Different Health Care Providers}

Objective - To detect differences in the prevalence of dyslipidemia in adult ambulatory laboratory tests from three different health care providers, in Salvador-Brazil, 1995.

Methods - The study was carried out using a probabilistic sample of 24 out of 104 laboratories $(\cong 25 \%)$ which performed the same enzymatic method for lipid tests with the same quality control. Laboratory tests results were separated into three groups, according to health care provider: Sistema Único de Saúde (SUS, governamental), Health Maintenance Organizations (HMO), and Private or Patients Health Insurance (PHI). Criteria for dyslipidemias in mg/dL were: total cholesterol $\geq 240 ; L D L$ $c \geq 160 ; H D L-c<35$ and triglycerides $\geq 200$. Prevalence rates, $95 \%$ confidence intervals $(C I)$ and $\chi^{2}$ test were used in the analysis.

Results - From the 5464 adult subjects, $14.8 \%$ were from SUS, 31.6\%. from HMO and 53.6\% PHI. The highest prevalence for hypercholesterolemia - 28.0\% (CI 24.0; 32.0) and for $L D L-c \geq 160 \mathrm{mg} / \mathrm{dL}-30.4 \%$ (CI 27.6; 33.2), were found in the HMO group. PHI had the highest prevalence for $H D L-c<35 \mathrm{mg} / \mathrm{dL}-12.3 \%$ (CI 10.0; 13.8), and for hypertrygliceridemia - 17.8\% (CI 16.3; 19.3). Most of the differences among health providers were statistically significant.

Conclusion - The best lypid profile observed in subjects from SUS suggests social differences in the prevalence of dyslipidemias. As compared to other dyslipidemias, $H D L-c<35 \mathrm{mg} / d L$ prevalence was lower than expected in all three groups. The data may provide insights to medical doctors and other health care professionals regarding the questions of dyslipidemias. It can also provide objective information to the patients and encourage them to change their life styles.

Key words: dyslipidemia, health care

Arq Bras Cardiol, volume 70 (nº 5), 331-335, 1998

Universidade Federal da Bahia - Salvador. Apoio CNPq

Correspondência: Ines Lessa - Parque Lucaia, QE - lote 03 (Rua Barachisio Lisboa) - 40295-120 - Salvador, BA

Recebido para publicação em 2/1/98

Aceito em 18/2/98
São raros os estudos sobre dislipidemias no Brasil e a maioria procede de casuísticas com doença ou suspeita de doença arterial coronária (DAC) ${ }^{1-4}$. Os estudos populacionais são ainda mais restritos, baseados em pequenas 
amostras ou grupos específicos de trabalhadores ${ }^{5-7}$. No início da década de 80, em Salvador, a freqüência de hipercolesterolemia nos casos incidentes de infarto agudo do miocárdio, com colesterol dosado na fase aguda da doença, era de cerca de $41 \%{ }^{8}$. Embora populacional e de incidência, o desenho deste estudo não permitia a obtenção da freqüência do colesterol elevado nos não casos.

Em populações de outros países, a prevalência tende a ser elevada, com variações geográficas na sua magnitudes ${ }^{9-11 .} \mathrm{O}$ único estudo brasileiro, que incluiu várias capitais, revelou diferenças regionais importantes nos níveis dos lípides séricos, particularmente do HDL-c em pacientes com $\mathrm{DAC}^{3}$. Os investigadores nacionais que mencionam nível socioeconômico citam freqüências mais elevadas de hipercolesterolemia ou de outra dislipidemia nos grupos com melhor nível social ${ }^{5,6}$.

A inexistência de estudos que representem a população geral em qualquer parte do Brasil deve-se, principalmente, a duas razões: a) falta de recursos financeiros para investigações populacionais e b) carência de pesquisadores em epidemiologia das doenças crônicas não-transmissíveis e seus determinantes. Por estas razões, o uso racional de bancos de dados secundários deve ser utilizado em algumas situações, sendo uma delas as dislipidemias. Isso foi feito em Salvador, 1995, onde, com base em casuística representativa da demanda laboratorial não-hospitalar da cidade, detectaram-se prevalências de dislipidemias muito elevadas em adultos dos dois sexos ${ }^{12} \mathrm{e}$, excepcionalmente elevadas, em mulheres acima dos 50 anos, excetuando-se sempre o HDL-c $<35 \mathrm{mg} / \mathrm{dL}^{12}$. Em seqüência ao referido estudo, o objetivo desta parte, baseada no mesmo banco de dados e metodologia, é de detectar diferenças nas prevalências das dislipidemias em adultos da demanda laboratorial de três diferentes prestadores de assistência médica.

\section{Métodos}

O estudo das dislipidemias na cidade do Salvador corresponde a um projeto integrado de pesquisa, planejado para utilização de banco de dados secundários. Por se tratarem de dados de diversos laboratórios, foram tomados os cuidados necessários para evitar vieses de natureza diversificada, descritos em outros trabalhos, um deles apresentado no LIII Congresso da Sociedade Brasileira de Cardiologia, $1997^{13}$ e os demais jámencionados naintrodução.
Estudo de corte transversal, clínico epidemiológico que utiliza resultados de dosagens dos lípides séricos de uma amostra probabilística de 25\% dos 104 laboratórios de análises clínicas de Salvador, 1995, que usavam o mesmo método enzimático e o controle de qualidade da Sociedade Brasileira de Patologia Clínica. Os meses ímpares foram sorteados para o estudo. O "n" total correspondeu a 10.192 pessoas das quais 9.723 eram adultos com idades $\geq 20$ anos, atendidos em 24 dos 26 laboratórios amostrados. Houve recusa de um laboratório e em outro o arquivo era descartado a cada três meses. A fonte prestadora de assistência contava com 5.464 indivíduos $(53,6 \%)$, distribuídos segundo as fontes, em: procedentes do Sistema Único de Saúde (SUS), dos convênios (C) e dos seguros privados de saúde + particulares (SP). A demanda do SUS, em Salvador, é toda procedente dos estratos sociais mais baixos; a dos C é de difícil classificação, mas com predominância do estrato médio e os SP englobam, predominantemente, os estratos médio a alto.

Os critérios para diagnóstico das dislipidemias foram o do II Consenso Brasileiro sobre Dislipidemias ${ }^{14}$. Foram realizadas análises pelas determinações das prevalências, seus intervalos de confiança (IC) a $95 \%$ e pelos testes estatísticos do $\chi^{2}$ e de diferenças entre duas proporções.

\section{Resultados}

Das 5.464 pessoas, $808(14,8 \%)$ foram encaminhadas pelo SUS, $1.726(31,6 \%)$ pelos convênios e $2.930(53,6 \%)$ pelos SP. Níveis limítrofes do colesterol e do LDL-c, bem como as prevalências de hipercolesterolemia e de LDL-c anormal foram mais elevados para o grupo de conveniados. A maior prevalência de hipercolesterolemia neste grupo diferiu significantemente dos demais $(\mathrm{p}<0,01)$. OLDL-c elevado entre conveniados diferiu em relação aos pacientes dos $\mathrm{SP}(\mathrm{p}<0,001)$. A diferença entre SP e SUS também foi estatisticamente significante (tab. I e II). A prevalência do HDL-c anormal foi mais elevada para pessoas procedentes dos SP, com $\mathrm{p}<0,05$ na comparação com SUS e $<0,01$ na comparação com C. Prevalência mais baixa de HDL-c $<35 \mathrm{mg} / \mathrm{dL}$ foi detectada para conveniados. Esse grupo não diferiu estatisticamente do grupo SUS (tab. III). Hipertrigliceridemia foi também mais prevalente na demanda dos SP, seguindo-se os conveniados, com diferenças significantes em relação à demanda do SUS ( $p<0,01 \mathrm{e}<0,02$, respectivamente) (tab. IV), sem diferença entre SP e C $(\mathrm{p}>0,05)$. Todos os IC das

\begin{tabular}{|c|c|c|c|c|c|c|c|c|}
\hline \multirow[b]{2}{*}{ Colesterol total } & \multicolumn{2}{|c|}{ SUS } & \multicolumn{2}{|c|}{$\mathrm{C}$} & \multicolumn{2}{|c|}{ SP } & \multicolumn{2}{|c|}{ Total } \\
\hline & $" n "$ & $\%$ & "n" & $\%$ & "n" & $\%$ & $" n "$ & $\%$ \\
\hline$<200$ & 374 & 46,3 & 671 & 39,0 & 1303 & 44,5 & 2348 & 40,2 \\
\hline $200-239$ & 238 & 29,4 & 569 & 33,0 & 930 & 31,8 & 1737 & 29,7 \\
\hline$\geq 240$ & 188 & $23,3 *$ & 483 & $28,0 * *$ & 692 & $23,7^{+}$ & 1763 & $30,1^{++}$ \\
\hline NR & 8 & 1,0 & 3 & & 5 & & 16 & \\
\hline Total & 808 & 100,0 & 1726 & 100,0 & 2930 & 100,0 & 5464 & 100,0 \\
\hline
\end{tabular}




\begin{tabular}{|c|c|c|c|c|c|c|c|c|}
\hline \multirow[b]{2}{*}{ LDL-c } & \multicolumn{2}{|c|}{ SUS } & \multicolumn{2}{|c|}{ C } & \multicolumn{2}{|c|}{ SP } & \multicolumn{2}{|c|}{ Total } \\
\hline & $\mathrm{N}^{\circ}$ & $\%$ & $\mathrm{~N}^{0}$ & $\%$ & $\mathrm{~N}^{\circ}$ & $\%$ & $\mathrm{~N}^{\circ}$ & $\%$ \\
\hline$<130$ & 124 & 49,0 & 457 & 43,2 & 821 & 51,2 & 1402 & 48,1 \\
\hline $130-159$ & 58 & 22,9 & 279 & 26,4 & 394 & 24,6 & 731 & 25,1 \\
\hline$\geq 160$ & 71 & $28,1 *$ & 322 & $30,4^{* *}$ & 387 & $24,2^{+}$ & 780 & $26,8^{++}$ \\
\hline NR & 555 & & 668 & & 1328 & & 2551 & \\
\hline Total & 808 & 100,0 & 1726 & 100,0 & 2930 & 100,0 & 5464 & 100,0 \\
\hline
\end{tabular}

\begin{tabular}{|c|c|c|c|c|c|c|c|c|}
\hline \multirow[b]{2}{*}{ HDL-c } & \multicolumn{2}{|c|}{ SUS } & \multicolumn{2}{|c|}{ C } & \multicolumn{2}{|c|}{ SP } & \multicolumn{2}{|c|}{ Total } \\
\hline & $\mathrm{N}^{\circ}$ & $\%$ & $\mathrm{~N}^{\circ}$ & $\%$ & $\mathrm{~N}^{\circ}$ & $\%$ & $\mathrm{~N}^{\circ}$ & $\%$ \\
\hline$<35$ & 25 & $8,3^{*}$ & 89 & $6,6^{* *}$ & 232 & $12,3^{+}$ & 346 & $9,8^{++}$ \\
\hline$\geq 35$ & 278 & 91,7 & 1252 & 94,4 & 1644 & 87,7 & 3174 & 90,2 \\
\hline NR & 505 & - & 385 & & 1054 & & 1944 & \\
\hline Total & 808 & 100,0 & 1726 & 100,0 & 2930 & 100,0 & 5464 & 100,0 \\
\hline
\end{tabular}

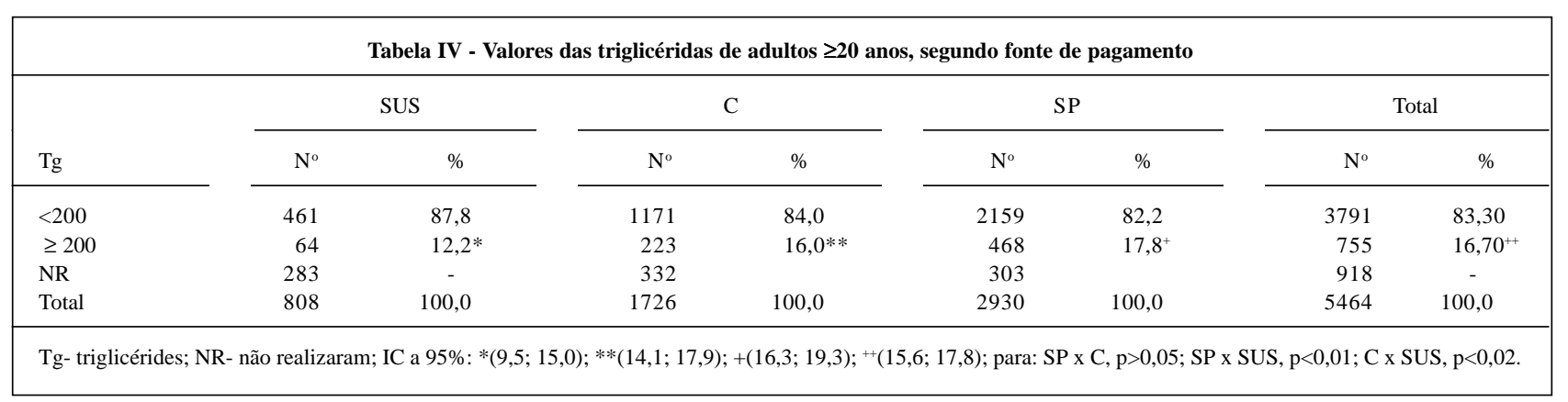

\begin{tabular}{|lrrrr|}
\hline \multicolumn{5}{|c|}{$\begin{array}{c}\text { Tabela V } \\
\text { - Número estimado de pessoas da amostra com provável } \\
\text { dislipidemia não }\end{array}$} \\
diagnosticada* \\
\hline Dislipidemia & SUS & C & SP & Total \\
\hline Col total $\geq 240 \mathrm{mg} / \mathrm{dL}$ & 2 & 1 & 1 & 4 \\
HDL-c $<35 \mathrm{mg} / \mathrm{dL}$ & 42 & 25 & 131 & 198 \\
LDL-c $\geq 160 \mathrm{mg} / \mathrm{dL}$ & 156 & 210 & 321 & 687 \\
Tg $\geq 200 \mathrm{mg} / \mathrm{dL}$ & 34 & 53 & 54 & 141 \\
\hline Tg- triglicérides; Col- colesterol; *base para os cálculos: prevalências das \\
dislipidemias em cada grupo e número de pessoas que não realizou o exame.
\end{tabular}

prevalências foram de pequena amplitude. A figura 1 resume as prevalências observadas nas quatro tabelas.

Excluindo-se o LDL-c, que pode ser estimado pelo profissional solicitante, o número de pessoas com hipertrigliceridemia ou HDL-c baixo que perdeu a oportunidade do diagnóstico à época do exame foi expressivo (tab. V).

\section{Discussão}

Não existem estudos sobre dislipidemias que levem em consideração análise por prestador de assistência. Ou-

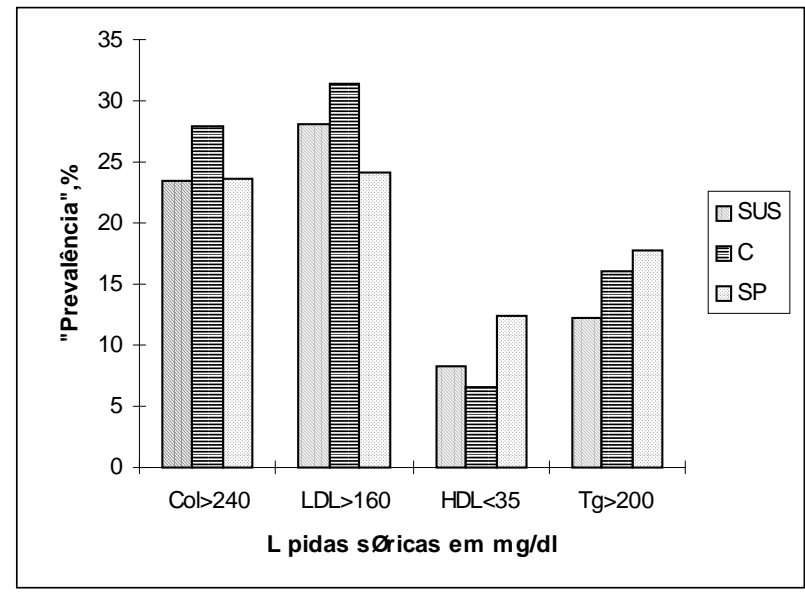

Fig. 1 - Prevalências de dislipidemias por prestador de assistência.

tros marcadores sociais foram usados por alguns autores brasileiros: Rouquayrol e $\mathrm{col}^{5}$ encontraram maior prevalência de hipercolesterolemia entre moradores de mansões do que entre favelados de Fortaleza; em Cotia, (SP), Martins e $\mathrm{col}^{6}$ relataram níveis séricos mais elevados para o coles- 
terol e triglicérides em classes sociais mais elevadas; para funcionários de hospitais universitários, a prevalência de hipercolesterolemia foi de $26 \%$ no Rio de Janeiro ${ }^{7}$.

O laboratório, como banco de dados secundários, impossibilita determinar prevalências por estratos sociais. Contudo, a classificação da demanda, por prestador de assistência, permite apenas afirmar que os indivíduos dos estratos sociais mais baixos são, na quase totalidade, atendidos pelo SUS. Excetuando-se o HDL-c $<35 \mathrm{mg} / \mathrm{dL}$, as demais dislipidemias foram de elevada prevalência em Salvador, nenhuma delas no grupo do SUS. Apenas para o LDL-c e HDL-c, o grupo do SUS, comparado aos demais prestadores de assistência, ocupou a segunda posição. Se a equivalência entre usuários do SUS e estrato social é verdadeira - o que não parece duvidoso -, os dados de Salvador concordam com os de Rouquayrol e col $^{5}$ e com os de Martins e $\mathrm{col}^{6}$. O grupo SUS também apresentou as menores frequiências de níveis séricos limítrofes para o colesterol total e para oLDL-c.

Para a demanda dos convênios, é difícil a distinção de estratos sociais (estrato misto). Como as entidades conveniadas são representadas por diversos setores da economia (indústria, comércio e serviços), os convênios destinam-se, sobretudo, aos indivíduos na faixa etária economicamente ativa e seus dependentes. Inclui proporções baixas dos estratos sociais extremos, com maioria de componentes do estrato médio. Foi na demanda dos convênios que se observaram as mais elevadas taxas de prevalência de hipercolesterolemia e do LDL-c $\geq 160 \mathrm{mg} / \mathrm{dL}$, ambas com diferenças significantes em relação ao grupo SP; para o colesterol total também houve diferença significante em relação ao grupo SUS. Ressalta-se que níveis séricos do LDL-c elevado é fator de risco mais freqüentemente associado à DAC no homem do que da mulher, reduzindo-se as diferenças com o avanço da idade destas ${ }^{15,16}$. Considerando isoladamente o LDL-c, componentes do grupo convênios apresentam maior risco do que os demais.

Em 1991, 25\% da população brasileira era atendida pelos seguros privados de saúde ${ }^{16}$. Considerando que esse grupo compõe-se de indivíduos que podem assumir mais um encargo financeiro com um plano ou seguro privado de saúde, sem isenção do pagamento das taxas para o Instituto Nacional da Seguridade Social, presume-se que represente, além de parcela do estrato médio, a grande maioria dos indivíduos dos estratos sociais mais diferenciados. Esclarecese, no entanto, que vários setores da economia também contratam seguros privados para assistência aos seus trabalhadores. Foi na demanda dos SP que se detectaram as maiores prevalências de hipertrigliceridemia e do HDL-c $<35 \mathrm{mg} / \mathrm{dL}$. Das dislipidemias, essa é a que se configura como a de maior risco cardiovascular para as mulheres ${ }^{15,16}$.

As pequenas amplitudes dos intervalos de confiança demonstram precisão no tamanho da amostra de cada grupo. Este dado, associado aos cuidados metodológicos adotados, possibilitam inferência para prática médica similar. Para a própria amostra, pode-se estimar, para cada prestador de assistência, o número de casos de dislipidemias não diagnosticado. Esse número só foi desprezível para o colesterol total.

Conquanto não haja obrigatoriedade da solicitação de todo o perfil lipídico na prática médica cotidiana, o seu custo é tão reduzido que muitas pessoas poderiam ter o perfil lipídico solicitado com a periodicidade recomendada nos programas preventivos, a depender da idade e do perfil de risco ou doenças presentes. A probabilidade de resultados anormais para os lípides séricos é muito mais elevada do que o comumente visto em solicitações procedentes da mesma prática médica para exames complementares de alto custo ${ }^{18,19}$, freqüentemente baseados em queixas habituais, não exploradas clinicamente e com alta probabilidade de resultados normais ${ }^{19}$.

Se os resultados demonstrados não podem ser inferidos para a população geral, nem para estratos sociais, eles representam a demanda laboratorial da prática médica cotidiana de Salvador, para a qual o estudo foi planejado e foi capaz de detectar diferenças nas prevalências das dislipidemias pela procedência da demanda, segundo a fonte pagadora dos exames. Com baixo custo foi possível mostrar a necessidade do incentivo a programas educativos para o de controle das dislipidemias, inexistentes na saúde pública do país.

\section{Agradecimentos}

À todos os profissionais proprietários dos laboratórios de análises clínicas de Salvador que colaboraram fornecendo seus bancos de dados.

\section{Referências}

1. Luz PL, Carvalho MEA, Cardoso RHA, Gallucci SDB, Pieligi F. - Incidência de dislipidemias e sua relação com doença arterial coronária em populações brasileiras. Arq Bras Cardiol 1990; 54: 257-64.

2. Giannini SD, Deveriacki BE, Góis JM, Diament J et al - Prevalência de dislipidemias primárias em indivíduos com e sem história familiar de coronariopatia, tendo como referência os valores do National Cholesterol Education Program (NCEP). Arq Bras Cardiol 1992; 58: 281-7.

3. Forti N, Fukushima J, Giannini SD - Perfil lipídico de indivíduos submetidos à cinecoronariografia em diferentes regiões do Brasil. Arq Bras Cardiol 1997; 68:333-42.

4. Ladeia AM, Guimarães AC, Lima JC - Perfil lipídico e doença arterial coronária. Arq Bras Cardiol 1994; 63: 101-6.

5. Rouquayrol MZ, Veras FMF, Vasconcelos J, Bezerra RC, Gomes ILP, Bezerra, FAF
- Fatores de risco em doença coronária. Inquérito epidemiológico em estratos habitacionais de um bairro de Fortaleza. Arq Bras Cardiol 1987; 49: 339-47.

6. Martins IS, Coelho LT, Matos IMS, Mazzilli RN, Trigo M, Wilson D Dislipidemias e alguns fatores de risco associados em uma população periférica da região metropolitana de São Paulo, SP-Brasil. Um estudo piloto. Rev Saúde Públ S Paulo 1989; 23: 236-43.

7. Nogueira AR, Alves PM, Miranda RF, Boechat NL-Colesterol e outros fatores de risco cardiovascular em servidores da UFRJ. Prevalências e influências sociais. Arq Bras Cardiol 1990; 55: 227-32.

8. Lessa I, Cortes E, Souza F J, Pondé Netto J, Almeida FA-Epidemiology of acute myocardial infarction in Salvador, Brazil. I-Incidence, lethality and mortality. Pan Am Health Organiz Bull 1987; 21: 28-37. 
9. Heiss G, Tammir I, Davis CE et al - Lipoprotein-cholesterol distributions in selected North-American populations: the Lipid Research Clinics Program Prevalence Study. Circulation 1980; 61: 302-15.

10. Posadas RC, Tapia CR, Lerman GI et al - Cholesterol leavels and prevalence of hypercholesterollemia in a Mexican adult population. Atherosclerosis 1995; 118: 275-84.

11. Hodge AM, Dowse GK, Erasmus RT et al - Serum lypids and modernization in coastal and highland Papua new Guinea. Am J Epidemiol 1996; 144: 1129-42.

12. Lessa I, Conceição Filho JL, Souza ML, Oliveira V, Carneiro J Melo- Prevalência de dislipidemias em adultos da demanda laboratorial de Salvador. Brasil Arq Bras Cardiol 1997; 69: 395-400.

13. Grupo de estudo das dislipidemias em Salvador, Bahia - Dislipidemias em diferentes grupos sociais da demanda laboratorial não-hospitalar de Salvador. Arq Bras Cardiol. 1997; (69 supl I): 98.

14. Sociedade Brasileira de Cardiologia - II Consenso Brasileiro Sobre
Dislipidemias. Detecção, Avaliação e Tratamento. Arq Bras Cardiol 1996; 67: 113-8.

15. Crouse JR - Gender, lipoprotein, diet and cardiovascular risk. Lancet 1989; 1 : 318-20.

16. Corrao JM, Becker RC, Ockene IS, Hamilton GA-Coronary heart disease risk factors in women. Cardiology 1990; 77(suppl 2): 8-24.

17. Buss PM - Assistência hospitalar no Brasil, 1984-1991: uma análise preliminar baseada no Sistema de Informação Hospitalar do SUS. Inf. Epidemiol.(SUS, CENEPI, Ministério da Saúde) 1993; 2: 5-42.

18. Oliveira ATR, Rabelo LM, Lessa I - Características da demanda por tomografia computadorizada crânio-encefálica em Salvador: motivos e custos dos exames. Arq Bras Neuro-Psiquiat S. Paulo 1992; 50:91-8.

19. Argollo N - Epidemiologia dos tumores cerebrais na infância no Estado da Bahia: método da captura-recaptura. Dissertação de Mestrado, Faculdade de Medicina, Universidade Federal da Bahia, 1997 